\title{
Increased risk of malignancy in patients with systemic lupus erythematosus: population- based cohort study in Korea
}

\author{
Jung-Yong Han ${ }^{1}$, Hyoungyoung Kim', Sun-Young Jung ${ }^{2}$, Eun Jin Jang ${ }^{3}$, Soo-Kyung Cho ${ }^{1}$ and \\ Yoon-Kyoung Sung ${ }^{1 *}$
}

\begin{abstract}
Background: This study aimed to evaluate the crude incidence rates and relative risk of malignancy in Korean patients with SLE.

Methods: We conducted a retrospective nationwide cohort study using databases from the National Health Insurance Service in Korea. All prevalent SLE patients aged over 19 were identified from January 2012 to December 2014 and observed until the diagnosis of malignancy, death, or end of the study, December 2015. The crude incidence rates (IRs) and standardised incidence ratios (SIRs) of overall and site-specific malignancies in SLE patients were estimated.

Results: We identified 17,854 SLE patients and during the observation period (60,511 person-years [PYs]), 768 solid malignancies (126.9/10,000 PYs) and 68 haematologic malignancies (11.2/10,000 PYs) occurred in SLE patients. In SLE patients, breast and reproductive system and thyroid cancers occurred predominantly, followed by liver and colon cancers. The SIRs of overall, solid, and haematologic malignancies of SLE patients compared to the general population were 1.8 (95\% confidence interval [Cl] 1.6-1.9), 1.7 (95\% Cl 1.5-1.8), and 5.9 (95\% Cl 4.8-7.3), respectively. In solid malignancies, head and neck (2.7, 95\% Cl 1.1-4.2), bladder (2.4, 95\% Cl 1.1-3.8), liver (1.9, 95\% Cl 1.4-2.3), pancreas (1.9, 95\% Cl 1.3-2.6), lung (1.8, 95\% Cl 1.2-2.4), colon (1.7, 95\% Cl 1.3-2.2), thyroid (1.6, 95\% Cl 1.3-1.8) and breast and reproductive system $(1.5,95 \% \mathrm{Cl} 1.2-1.7)$ cancers are at increased risk in SLE patients.
\end{abstract}

Conclusion: An increased risk of haematologic and solid malignancies was observed in Korean patients with SLE compared to the general population.

Keywords: Systemic lupus erythematosus, Malignancy, Incidence rate, Standardised incidence ratio

\section{Background}

Systemic lupus erythematosus (SLE) is a relatively common systemic autoimmune rheumatic disease with a wide range of clinical presentations resulting from the involvement of multiple organ systems [1-5]. It commonly affects young and middle-aged people and is

\footnotetext{
*Correspondence: sungyk@hanyang.ac.kr

${ }^{1}$ Department of Rheumatology, Hanyang University Hospital for Rheumatic Diseases, Seoul, South Korea

Full list of author information is available at the end of the article
}

characterised by a 9:1 female to male ratio of disease incidence [6]. Although survival in SLE has improved during the past 30 years, its mortality remains high relative to the general population, and significant sex, racial, and regional disparities persist $[7,8]$.

The risk of malignancy in SLE has become an increasing concern because cumulative immunosuppressant drugs may be associated with increased cancer risk [9]. There is increasing evidence to suggest that patients with SLE have a higher overall risk of malignancy [10]. A 
recent multisite international cohort study reported an association between SLE and cancer (standardised incidence ratio, SIR, 1.14, 95\% CI 1.05-1.23), highlighting the risk for non-Hodgkin lymphoma (NHL) and leukaemia, but also demonstrating an increased risk of vulvar, lung, thyroid and possibly liver cancers. On the other hand, it was reported that patients with SLE appear to have a decreased risk of breast, endometrial and ovarian cancers [11]. However, another comprehensive meta-analysis established epidemiologic evidence to support an association between SLE and increased risk for 16 cancers, except for prostate cancer and cutaneous melanoma [12].

Evidence of relationships between cancer and SLE has been provided in some studies of Korean populations [13, 14]. SLE patients were found to have more risk of malignancy, such as cervical cancer, lymphoma and bladder cancer. However, the subjects in these previous studies were patients selected from specific centres, not a population-based nationwide level.

We therefore evaluated the incidence rates and relative risk of malignancy in all Korean patients with SLE compared to the general population in this study.

\section{Methods}

\section{Data sources}

\section{National claims database of Korea for SLE patients}

The Health Insurance Review and Assessment Service (HIRA) database, which includes the Korean population of around 50 million individuals, was the primary data source for this study. The HIRA database contains information about individual beneficiaries, and healthcare service information such as diagnosis, procedure, prescription and diagnostic tests from in- and outpatient care.

\section{National Health Insurance Service-National Sample Cohort} Database (NHIS-NSC) for the general population

NHIS has developed a National Sample Cohort (NSC) database using stratified random sample for age, gender, participants' eligibility stats, region and income level. A representative sample cohort of 1,025,340 participants, comprising $2 \%$ of the total Korean population, was included in this study [15].

\section{Study populations \\ Patients with SLE}

We identified all of the Korean patients with SLE who were registered in the rare intractable disease (RID) registration programme in Korea. All patients in the programme had to have received a specific RID code based on the 1997 Update of the 1982 American College of Rheumatology Revised Criteria for Classification of Systemic Lupus Erythematosus [16]. Fulfilment of the criteria is reviewed strictly by the healthcare institution, because patients registered in this programme were supported financially by government aid.

Using this HIRA database, patients with both an ICD10 code (M32.0) and RID code (V136) were defined as SLE patients. All the prevalent SLE patients aged $\geq 19$ years registered in the RID programme between 2012 and 2014 were included in our study. Patients who had other connective tissue diseases such as rheumatoid arthritis or Sjögren's syndrome were excluded. To identify incident malignancy cases, we excluded patients who had a history of any malignancy in the 12 months before the index date (Fig. 1).

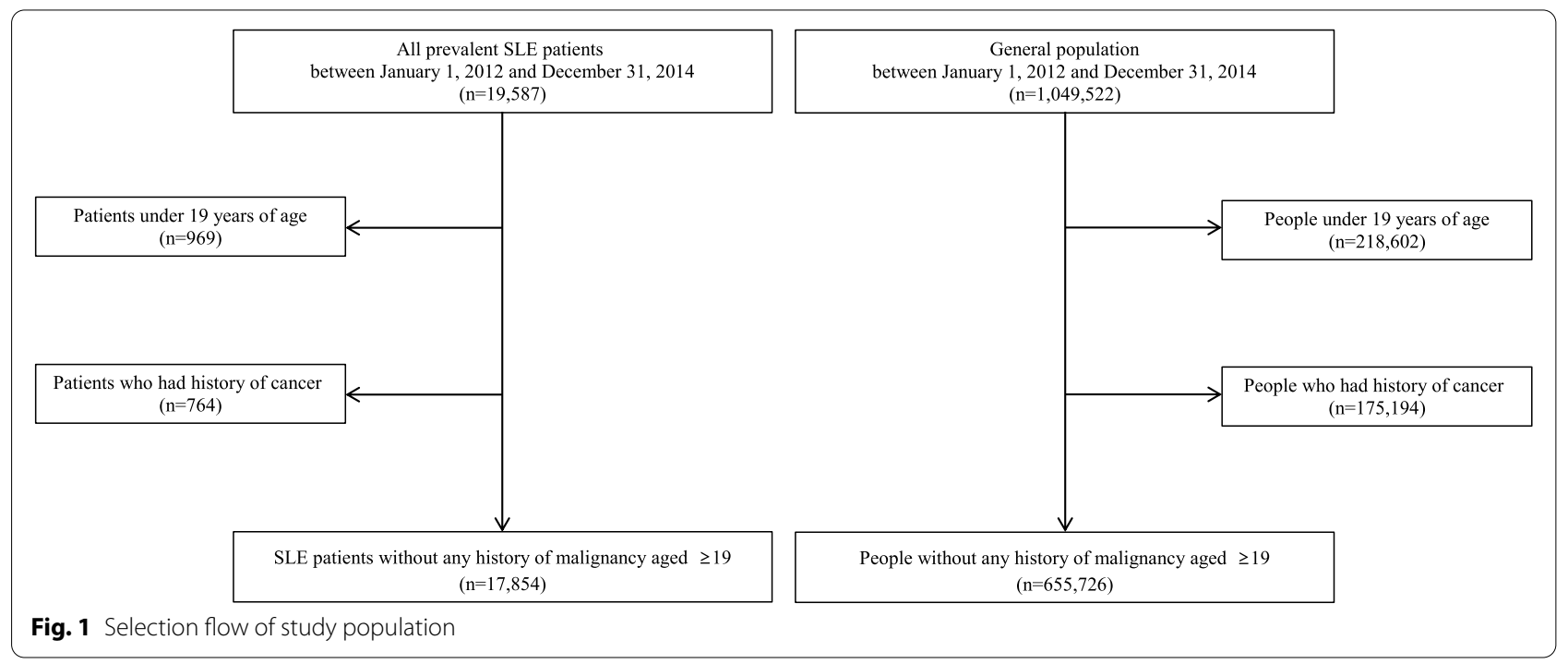




\section{General population as a control group}

As a comparator, we recruited individuals in the general population who had any medical claims from the NHIS-NSC database between 2012 and 2014 with matching the calendar years. After that, we excluded the people aged under 19 and those who had claimed any malignancy 12 months before the index date.

\section{Definition of incident malignancy}

The occurrence of malignancy was regarded as the first claims under ICD-10 codes, such as C00 to C97, including both solid and haematologic malignancies according to the type or site of malignancy. Among solid malignancies, breast, cervix, corpus ovary, prostate and testis malignancies were grouped as breast and reproductive system malignancies and specific codes for each malignancy were masked. We set the date of the first claims of each malignancy as the date that the diagnosis of the malignancy was made. In cases where several malignancies occurred at different time points, we regarded every malignancy as independent incident cases during the observations [17].

The index date was defined as the date of the first claim for diagnosis with SLE combined with RID code or first claim of the control group for the observation period from 2012 to 2014. The previous one year before the index date was defined as the history period. Patients were followed from the index date to the date of malignancy diagnosis, death, or the end of 2015 if any malignancy was not diagnosed. If the patients were diagnosed with multiple types of malignancies, the follow-up period was established with the date of the last diagnosis of any type of malignancy.

\section{Statistical analysis}

We calculated the crude incidence rate (IR) of malignancies per 10,000 person-year (PYs) in patients with SLE. Crude IR was calculated by dividing the incident cases of malignancies by the total observational periods of patients with a $95 \%$ confidence interval (CI). The crude IR of overall and each malignancy according to gender was presented in the descriptive analysis.

To estimate the risk of malignancy in patients with SLE compared to the general population, we calculated standardised incidence ratios (SIRs) by dividing the observed number of malignancies of SLE patients by the expected number of malignancies calculated from the accumulated PYs and age, gender, and calendar period-specific malignancy IRs of the general population. The SIR was described with 95\% CI. All statistical
Table 1 Baseline characteristics of study population

\begin{tabular}{|c|c|c|}
\hline Variables & $\begin{array}{l}\text { SLE patients } \\
(n=17,854)\end{array}$ & $\begin{array}{l}\text { General population } \\
(n=649,010)\end{array}$ \\
\hline Age, years & $42.3 \pm 13.2$ & $44.2 \pm 16.1$ \\
\hline Sex, female & $16,143(90.4)$ & $306,649(47.2)$ \\
\hline Duration of observation, years & $3.4 \pm 0.9$ & $3.7 \pm 0.6$ \\
\hline \multicolumn{3}{|l|}{ Payer type } \\
\hline National health insurance & $16,752(93.8)$ & $630,446(71.1)$ \\
\hline Medical aid & $1102(6.2)$ & $18,564(2.9)$ \\
\hline \multicolumn{3}{|l|}{ Type of institution } \\
\hline Tertiary hospital & $12,362(69.2)$ & $15,059(2.3)$ \\
\hline General hospital & $3837(21.5)$ & $30,927(4.8)$ \\
\hline Community hospital & $146(0.8)$ & $36,687(5.6)$ \\
\hline Clinic & $1377(7.7)$ & $239,369(36.9)$ \\
\hline Other & $132(0.7)$ & $326,968(50.4)$ \\
\hline Number of comorbidities & $1.2 \pm 0.6$ & $0.1 \pm 0.3$ \\
\hline \multicolumn{3}{|l|}{ Comorbidity } \\
\hline Myocardial infarction & $21(0.1)$ & $468(0.07)$ \\
\hline Congestive heart failure & $162(0.9)$ & $1437(0.2)$ \\
\hline Peripheral vascular disease & $512(2.9)$ & $4280(0.7)$ \\
\hline Cerebrovascular disease & $253(1.4)$ & $3733(0.6)$ \\
\hline Dementia & $38(0.2)$ & $132(0.02)$ \\
\hline Chronic pulmonary disease & $492(2.8)$ & $12,724(2.0)$ \\
\hline Peptic ulcer disease & $1084(6.1)$ & $10,119(1.6)$ \\
\hline Mild liver disease & $920(5.2)$ & $7558(1.2)$ \\
\hline Diabetes mellitus & $601(3.4)$ & $13,323(2.1)$ \\
\hline Hemiplegia or paraplegia & $51(0.3)$ & $685(0.1)$ \\
\hline Renal disease & $474(2.7)$ & $1,180(0.2)$ \\
\hline $\begin{array}{l}\text { Moderate or severe liver } \\
\text { disease }\end{array}$ & $11(0.1)$ & $157(0.02)$ \\
\hline Charlson Comorbidity Index score & $0.30 \pm 0.69$ & $0.10 \pm 0.40$ \\
\hline
\end{tabular}

Numerical quantitative data were presented by "mean \pm SD" and categorical data were presented by "frequency (\%)"

analyses were performed using SAS 9.2 (SAS Institute Cary, NC)

\section{Results}

\section{Baseline characteristics}

Among the 19,587 SLE patients extracted from the NHIS database, 969 patients under 19 years of age and 764 patients with a previous history of cancer were excluded. We finally identified 17,854 SLE patients and most of these were female (women, $n=16,143$ and men, $n=1,711$, 90.4\%).

Among 1,049,522 people from the general population in the NHIS-NSC database, we excluded 218,602 people under 19 years of age and 175,194 patients with a previous history of cancer. Finally, we included 649,010 people from the general population (women, $n=306,649$ and $m e n=342,361)$ in this analysis as a control group. 
Table 2 The crude incidence rate of malignancies in patients with SLE

\begin{tabular}{|c|c|c|c|c|c|c|}
\hline \multirow[t]{2}{*}{ Type of malignancy } & \multicolumn{2}{|c|}{ Total $(n=17,854)$} & \multicolumn{2}{|c|}{ Female $(n=16,143)$} & \multicolumn{2}{|c|}{ Male $(n=1711)$} \\
\hline & No. of cases & $\begin{array}{l}\text { Incidence per } \\
10,000 \text { PYs }(95 \% \mathrm{Cl})\end{array}$ & No. of cases & $\begin{array}{l}\text { Incidence per } \\
10,000 \text { PYs }(95 \% \mathrm{Cl})\end{array}$ & No. of cases & $\begin{array}{l}\text { Incidence per } \\
10,000 \mathrm{PYs}(95 \% \mathrm{Cl})\end{array}$ \\
\hline Overall malignancies & 836 & $138.16(128.79,147.52)$ & 738 & $134.45(124.75,144.15)$ & 98 & $174.40(139.87,208.93)$ \\
\hline Solid malignancy & 768 & $126.92(117.94,135.90)$ & 686 & $124.97(115.62,134.33)$ & 82 & $145.93(114.34,177.51)$ \\
\hline $\begin{array}{l}\text { Breast and reproductive } \\
\text { system }\end{array}$ & 175 & $28.92(24.64,33.21)$ & 164 & $29.88(25.30,34.45)$ & 11 & $19.58(8.01,31.14)$ \\
\hline Thyroid & 154 & $25.45(21.43,29.47)$ & 148 & $26.96(22.62,31.31)$ & 6 & $10.68(2.13,19.22)$ \\
\hline Liver & 63 & $10.41(7.84,12.98)$ & 55 & $10.02(7.37,12.67)$ & 8 & $14.24(4.37,24.10)$ \\
\hline Colon & 61 & $10.08(7.55,12.61)$ & 51 & $9.29(6.74,11.84)$ & 10 & $17.80(6.77,28.83)$ \\
\hline Lung & 34 & $5.62(3.73,7.51)$ & 25 & $4.55(2.77,6.34)$ & 9 & $16.02(5.55,26.48)$ \\
\hline Pancreas & 32 & $5.29(3.46,7.12)$ & 29 & $5.28(3.36,7.21)$ & 3 & $5.34(0,11.38)$ \\
\hline Stomach & 31 & $5.12(3.32,6.93)$ & 27 & $4.92(3.06,6.77)$ & 4 & $7.12(0.14,14.09)$ \\
\hline Bladder & 12 & $1.98(0.86,3.11)$ & 10 & $1.82(0.69,2.95)$ & 2 & $3.56(0,8.49)$ \\
\hline Head and neck & 11 & $1.82(0.74,2.89)$ & 9 & $1.64(0.57,2.71)$ & 2 & $3.56(0,8.49)$ \\
\hline Kidney & 10 & $1.65(0.63,2.68)$ & 5 & $0.91(0.11,1.71)$ & 5 & $8.90(1.10,16.70)$ \\
\hline Biliary tract & 7 & $1.16(0.30,2.01)$ & 4 & $0.73(0.01,1.44)$ & 3 & $5.34(0,11.38)$ \\
\hline $\begin{array}{l}\text { Brain and central nervous } \\
\text { system }\end{array}$ & 5 & $0.83(0.10,1.55)$ & 5 & $0.91(0.11,1.71)$ & 0 & NC \\
\hline Larynx & 2 & $0.33(0,0.79)$ & 2 & $0.36(0,0.87)$ & 0 & NC \\
\hline Haematology malignancy & 68 & $11.24(8.57,13.91)$ & 52 & $9.47(6.90,12.05)$ & 16 & $28.47(14.52,42.43)$ \\
\hline Non-Hodgkin lymphoma & 36 & $5.95(4.01,7.89)$ & 27 & $4.92(3.06,6.77)$ & 9 & $16.02(5.55,26.48)$ \\
\hline Multiple myeloma & 14 & $2.31(1.10,3.53)$ & 13 & $2.37(1.08,3.66)$ & 1 & $1.78(0,5.27)$ \\
\hline Leukaemia & 12 & $1.98(0.86,3.11)$ & 8 & $1.46(0.45,2.47)$ & 4 & $7.12(0.14,14.09)$ \\
\hline Hodgkin lymphoma & 3 & $0.50(0,1.06)$ & 3 & $0.55(0,1.16)$ & 0 & NC \\
\hline
\end{tabular}

$P Y$, person-year; $C l$, confidence interval; $N C$, not calculated

Baseline characteristics for SLE patients and the general population are shown in Table 1. Most patients with SLE were women (90.4\%) and younger than the general population. Compared to the general population, comorbidities were more common in patients with SLE (Table 1).

\section{Crude IR of malignancy in patients with SLE and the general population}

During the observation period (60,511 PYs), 836 overall malignancies (IR 138.16/10,000 PYs, 95\% CI 128.79147.52) occurred in SLE patients, compared with 22,735 events (IR 95.71/10,000 PYs, 95\% CI 94.46-96.95) in the general population $(2,375,523 \mathrm{PYs})$. In total, 768 incidents of solid malignancy (IR 126.9/10,000 PYs, 95\% CI 117.94-135.90) and 68 incidents of haematologic malignancy (IR 11.24/10,000 PYs, 95\% CI 8.57-13.91) occurred in SLE patients. For the number of sitespecific malignancy cases diagnosed in SLE patients, breast and reproductive system (crude IR 28.9, 95\% CI 24.6-33.2) has the highest IR, followed by thyroid (crude IR 25.4, 95\% CI 21.4-29.5), liver (crude IR 10.4,
95\% CI 7.8-12.9) and colon (crude IR 10.1, 95\% CI 7.612.6) (Table 2).

\section{Increased risk of malignancy in SLE patients compared to the general population}

Our study found SLE to be associated with increased risk of both solid (SIR 1.65, 95\% CI 1.53-1.77) and haematologic (SIR 5.87, 95\% CI 4.48-7.27) malignancies. For the risk of solid malignancy in SLE patients, head and neck cancer (SIR 2.7, 95\% CI 1.1-4.2) had the highest risk, followed by bladder (SIR 2.4, 95\% CI 1.1-3.8), liver (SIR 1.9, 95\% CI 1.4-2.3), pancreas (SIR 1.9, 95\% CI 1.3-2.6), lung (SIR 1.8, 95\% CI 1.2-2.4), colon (SIR 1.7, 95\% CI 1.3-2.2), thyroid (SIR 1.6, 95\% CI 1.3-1.8) and breast or reproductive system (SIR 1.5, 95\% CI 1.2-1.7) cancers. The risk of solid malignancy overall increased more in SLE patients than in the general population except for brain and central nervous system cancer (SIR 0.91, 95\% CI 0.11-1.72).

In regards to haematologic malignancies, SLE patients had a relatively high risk for multiple myeloma (SIR 8.19, 95\% CI 3.90-12.48), followed by NHL, (SIR 6.24, 95\% CI 4.20-8.27) and leukaemia (SIR 3.64, 95\% CI 1.58-5.70). However, the SIR of Hodgkin lymphoma (SIR 7.53, 95\% 


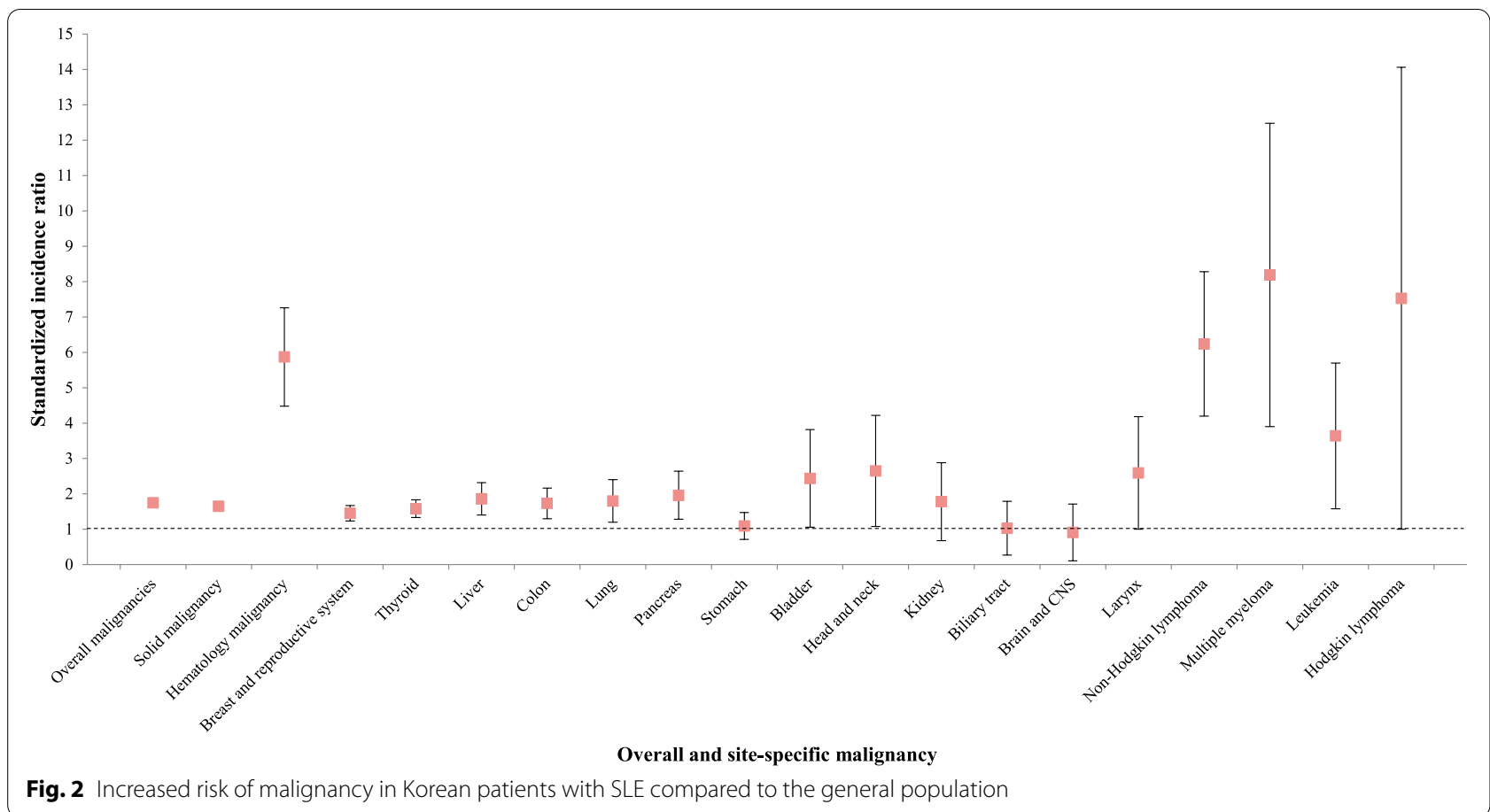

CI 1.00-16.05) was not significantly high in Korean patients with SLE (Fig. 2).

\section{Sex differences in the risk of malignancy}

There are some differences in the risk of malignancy between female and male SLE patients. Male SLE patients experienced higher crude IR of solid and haematologic malignancies than female SLE patients (Table 2). Female SLE patients had the highest crude IR of breast and reproductive system (crude IR 29.88/10,000PYs, 95\% CI 25.30-34.45) cancer, followed by thyroid (crude IR 26.96/10,000PYs, 95\% CI 22.62-31.31) and liver (crude 10.32/10,000PYs, 95\% CI 7.37-12.67) cancer. Otherwise, male SLE patients had the highest crude IR of reproductive system cancer including prostate cancer (crude IR 19.58/10,000PYs, 95\% CI 8.01-31.14), followed by colon (crude IR 17.80/10,000PYs, 95\% CI 6.77-28.83) and lung (crude IR 16.02/10,000PYs, 95\% CI 5.55-26.48) cancer and NHL (crude IR 16.02/10,000PYs, 95\% CI 5.55-26.48).

For the SIR of malignancy in female SLE patients compared to the general population, multiple myeloma (SIR 8.95, 95\% CI 4.08-13.82) was highest, followed by NHL (SIR 5.33, 95\% CI 3.32-7.34) and leukaemia (SIR 3.64, 95\% CI 1.58-5.70). In solid malignancy, bladder cancer (SIR 2.74, 95\% CI 1.04-4.44) had the highest incremental risk in female patients with SLE. Other solid malignancies such as thyroid, breast and reproductive system, liver, colon, lung and pancreas cancers also showed a higher risk in female patients with SLE than in the general population (Table 3 ).

In male SLE patients, the SIR of NHL (SIR 12.71, 95\% CI 4.41-21.02) was increased compared to the general population, but the risk of other haematologic malignancies such as leukaemia (SIR 7.80, 95\% CI 0.16-15.45) and multiple myeloma (SIR 3.90, 95\% CI 0-11.53) did not significantly increase. Although the risk of overall solid malignancies in male patients with SLE was increased (SIR 1.78, 95\% CI 1.39-2.16), the risk of site-specific solid cancers was not increased compared to the general population (Table 3).

\section{Discussion}

In this retrospective population-based cohort study, we found that individuals with SLE were at increased risk of haematologic malignancy as well as solid malignancy in Korean patients compared to the general population. Female patients with SLE had a significantly increased risk of multiple myeloma, NHL and solid cancers such as breast or reproductive system, thyroid, liver, colon, lung, pancreas and bladder cancers, whereas male patients with SLE demonstrated an increased risk of developing NHL.

Our results are similar to the profile observed in other studies $[12,18]$. A cohort study based on an international multi-centre showed an increased risk of NHL (SIR 4.39, 95\% CI 3.46-5.49) and cancers of the vulva (SIR 3.78, 95\% CI 1.52-7.78), lung (SIR 1.30, 95\% CI 1.04-1.60), thyroid 
Table 3 Sex differences in SIRs of malignancy in patients with SLE compared to general populations

\begin{tabular}{|c|c|c|c|}
\hline Type of malignancy & $\begin{array}{l}\text { Total } \\
(n=17,854)\end{array}$ & $\begin{array}{l}\text { Female } \\
(n=16,143)\end{array}$ & $\begin{array}{l}\text { Male } \\
(n=1711)\end{array}$ \\
\hline Overall malignancies & $1.75(1.63,1.87)$ & $1.72(1.60,1.84)$ & $2.05(1.65,2.46)$ \\
\hline Solid malignancy & $1.65(1.53,1.77)$ & $1.64(1.51,1.76)$ & $1.78(1.39,2.16)$ \\
\hline Breast and reproductive system & $1.45(1.23,1.66)$ & $1.43(1.21,1.65)$ & $1.76(0.72,2.81)$ \\
\hline Thyroid & $1.58(1.33,1.82)$ & $1.55(1.30,1.80)$ & $2.56(0.51,4.61)$ \\
\hline Liver & $1.86(1.40,2.32)$ & $1.98(1.46,2.50)$ & $1.32(0.40,2.23)$ \\
\hline Colon & $1.73(1.30,2.16)$ & $1.69(1.23,2.16)$ & $1.91(0.73,3.10)$ \\
\hline Lung & $1.80(1.20,2.41)$ & $1.73(1.05,2.41)$ & $2.03(0.70,3.35)$ \\
\hline Pancreas & $1.96(1.28,2.63)$ & $2.00(1.27,2.72)$ & $1.64(0,3.50)$ \\
\hline Stomach & $1.09(0.71,1.48)$ & $1.17(0.73,1.60)$ & $0.76(0.02,1.51)$ \\
\hline Bladder & $2.44(1.06,3.83)$ & $2.74(1.04,4.44)$ & $1.58(0,3.77)$ \\
\hline Head and neck & $2.65(1.08,4.21)$ & $2.71(0.94,4.47)$ & $2.41(0,5.75)$ \\
\hline Kidney & $1.78(0.68,2.89)$ & $1.06(0.13,1.99)$ & $5.54(0.68,10.39)$ \\
\hline Biliary tract & $1.03(0.27,1.80)$ & $0.69(0.01,1.37)$ & $3.00(0,6.39)$ \\
\hline Brain and central nervous system & $0.91(0.11,1.72)$ & $1.01(0.12,1.89)$ & $\mathrm{NC}$ \\
\hline Larynx & $2.59(1.00,6.17)$ & $5.02(0,11.98)$ & NC \\
\hline Haematology malignancy & $5.87(4.48,7.27)$ & $5.22(3.80,6.64)$ & $9.89(5.05,14.74)$ \\
\hline Non-Hodgkin lymphoma & $6.24(4.20,8.27)$ & $5.33(3.32,7.34)$ & $12.71(4.41,21.02)$ \\
\hline Multiple myeloma & $8.19(3.90,12.48)$ & $8.95(4.08,13.82)$ & $3.90(0,11.53)$ \\
\hline Leukaemia & $3.64(1.58,5.70)$ & $2.87(0.88,4.86)$ & $7.80(0.16,15.45)$ \\
\hline Hodgkin lymphoma & $7.53(1.00,16.05)$ & $9.41(0,20.06)$ & NC \\
\hline
\end{tabular}

The standardised incidence ratio (SIR) was presented with $95 \%$ confidence interval. $C l$, confidence interval; $N C$, not calculated

(SIR 1.76, 95\% CI 1.13-2.61) and possibly liver (SIR 1.87, 95\% CI 0.97-3.27) [11]. However, the risk of NHL, Hodgkin lymphoma and multiple myeloma with SIRs of 6.24 (95\% CI 4.20-8.27), 7.53 (95\% CI 1.00-16.05) and 8.19 (95\% CI 3.90-12.48), respectively, were estimated to be higher in our study than those in the international cohort study. In addition, the risk of cancer of the breast or reproductive system was increased in our study, whereas these risks were decreased in the other study [12]. Some of these differences in outcomes may be associated with the difference in either population factor such as ethnicity or lifestyle and the variation in the study design.

A nationwide cohort study performed in Taiwan using national claims database had the study design was quite similar to our study and Asian ethnicity. This study demonstrated an increased risk of vulva/vagina (SIR 4.76, 95\% CI 4.24-5.33), kidney (SIR 3.99, 95\% CI 3.74-4.27), nasopharynx (SIR 4.18, 95\% CI 3.93-4.45) and haematologic (SIR 4,96, 95\% CI 4.79-5,14) malignancies in SLE patients [19]. These were similar to our results, but some differences in site-specific risk in malignancies are made from the enrolment period and the relatively small sample size of the previous study.

We also compared our results with previous studies performed in Korea. A previous study in Korea has also reported that female SLE patients have an increased risk of overall malignancy (SIR 1.45, 95\% CI 0.74-.216) [14]. Inconsistent with our results, the published data described a decreased risk of thyroid cancer (SIR 0.98, 95\% CI 0.00-3.85) in female SLE patients, whereas our analysis showed an increased risk (SIR 1.55, 95\% CI 1.30-1.80). Otherwise, NHL was significantly increased by more than 15 -fold (SIR 15.2, 95\% CI 2.29-37.7), while our result shows 5.33 in SIR [14]. The reason for these differences is considered that the study collected from one single university hospital between 1997 and 2007. In addition, the sample size was less than 1000 , which was too small to ensure the external validity of the study results.

Recently, another study based on the NHIS database in Korea was performed [13]. It reported that Korean patients with SLE had a higher risk of overall malignancy (odds ratio, OR, 1.44; 95\% CI 1.327-1.559) and specific malignancies, such as cervical, thyroid, ovarian and oral cancer, as well as lymphoma, leukaemia and multiple myeloma, than controls. They used the same database for the selection of patients with SLE, but there were several differences compared to our study. We selected the NHIS-NSC database as a comparator and calculated the SIRs of each malignancy, whereas they used a retrospective cohort after 1:5 age and sexmatching. Hence, they could only present the increased 
risk with ORs. Definitions of inclusion and exclusion of SLE patients, wash-out period of malignancies and observational period of the study also differed from those of our study.

Despite some differences in the study design and data sources, the risk of overall malignancy in SLE patients has increased in all the studies. There could be various explanations for the aetiology and pathogenesis, though no clear mechanism has been established. First, immunosuppressive therapy could potentiate immune dysregulation and lead to developing cancer [20]. Some large-scale cohort studies have been conducted to identify the association between the development of malignancies and immunosuppressive agents $[18,21]$. In a multi-centre cohort study, the cumulative dose of cyclophosphamide affected the risk of malignancy in SLE patients [22]. Second, genomic studies have identified mutational genes at increased risk of malignancy in SLE patients. Chronic inflammation probably contributes to oxidative damage, DNA bases breaks and DNA-protein crosslinks [23]. In addition, SLE is associated with polymorphisms in DNA repair genes [24]. The combination of chronic inflammation and genetic predisposition to impaired DNA repair may contribute to DNA damage and increased risk of malignancy in SLE patients [25]. Third, in response to chronic immune damage, the cytokines that are associated with cell survival and proliferation, such as B cell activating factor, are overexpressed. Dysregulated immune proliferation could promote the translocation and juxtaposition of an oncogene beside a gene regulating immune function. This is related to the development of haematologic malignancies, particularly NHL [26]. Finally, some studies have reported an increased risk of reproductive system cancer, particularly vulvar and cervical cancer. Regarding the increased risk, sex hormonal effects and the altered clearance of human papillomavirus (HPV) are important factors [27].

Although the risk of malignancy seems to be increased, there was not a positive association between expected mortality and SLE in cohort studies. A cohort study that included 9547 patients with SLE from 23 sites worldwide, reported no increase in the mortality for overall malignancy (SMR 0.8, 95\% CI 0.6-1.0) [28]. A study of the mortality profile of SLE patients in France identified 1,593 deceased patients with SLE and reported an even lower SMR (0.40, 95\% CI 0.34-0.48) for overall malignancy [29]. These results indicate that other causes, such as cardiovascular disease, infectious disease or socioeconomic status, could attribute to the premature death of SLE patients.

This study has several strengths. First, the subjects in our study consisted of all the SLE patients in Korea and the general population through the NHIS database which provides a large nationwide and highly representative sample. We reduced environmental effects and prevented a possible ascertainment bias. Second, the study population and follow-up were well defined because of the broad coverage of the Korean NHIS. Third, through the estimation of not only the crude IR but also the SIR, we could determine the increased risk of malignancies in SLE patients compared to the general population.

Our study has several limitations. First, patients who are diagnosed with SLE are followed up and undergo regular examinations more closely than the general population. This is a surveillance bias that could increase the incidence of malignancy. Second, although our study showed increased cancer risk of SLE patients with statistical significance, a relatively short observation period may be noted in this study. Also, we could not obtain statistical significance in the malignancy risk of male SLE patients due to the relatively small size of subjects calculated. It should be considered that the duration and size of the study need to be extended. Third, we could not analyse the risk factors for the malignancy in patients with SLE, since we used the prevalence cohorts in both SLE patients and the general population. Further research which determines whether the development of cancer in patients with SLE is associated with the disease itself or drugs including immunosuppressive agents recently approved. To identify the risk factors for the development of malignancies, an incident cohort study may be conducted to minimise the immortal time bias [30].

Through this study, we established the site-specific cancer risk of Korean patients with SLE from the perspectives of frequency and of increasing risk compared to the general population. Further studies are needed to detect risk factors for the development of each malignancy and their impact on the mortality of SLE patients in Korea.

\section{Conclusion}

In conclusion, our study is a large-scale nationwide cohort study that supports evidence for increased risk of malignancy in SLE patients, not only in haematologic malignancies but also solid malignancies, compared to the general population.

\section{Abbreviation \\ SLE: Systemic lupus erythematosus; NHL: Non-Hodgkin lymphoma; HIRA: Health Insurance Review and Assessment Service; NHIS-NSC: National Health Insurance Service-National Sample Cohort Database; RID: Rare intractable disease; ICD: International Classification of Diseases; IR: Incidence rate; PYs: Person-years; Cl: Confidence interval; SIR: Standardised incidence ratio.}

\section{Acknowledgement}

None 


\section{Authors' contributions}

JYH and YKS contributed to the study design, data analyses, interpretation of results, and writing of the manuscript. HK and SKC contributed to the study design and interpretation of results. SYJ and EJJ contributed to the data analyses and interpretation of results. All authors read and approved the final manuscript.

\section{Funding}

This research was supported by a grant of the Korea Health Technology R\&D Project through the Korea Health Industry Development Institute (KHIDI), funded by the Ministry of Health \& Welfare, Republic of Korea (grant number: HI19C1202).

\section{Availability of data and materials}

The datasets used and/or analysed during the current study are available from the corresponding author on reasonable request.

\section{Declarations}

\section{Ethics approval and consent to participate}

As the databases extracted from NHIS could not be identified directly or through identifiers linked to the subjects, therefore, our study was exempted by the Institutional Review Board (IRB) of Hanyang University Hospital (IRB file NO. HYUH 2018-04-030).

\section{Consent for publication}

Not applicable

\section{Competing interests}

YKS has received research grants from Bristol-Myers Squibb, Eisai, Pfizer and JW Pharmaceutical. JYH, HK, SYJ, EJJ and SKC have nothing to declare.

\section{Author details}

${ }^{1}$ Department of Rheumatology, Hanyang University Hospital for Rheumatic Diseases, Seoul, South Korea. ${ }^{2}$ College of Pharmacy, Chung-Ang University, Seoul, South Korea. ${ }^{3}$ Department of Information Statistics, Andong National University, Andong, South Korea.

Received: 26 June 2021 Accepted: 12 October 2021

Published online: 27 October 2021

\section{References}

1. Ceccarelli F, Perricone C, Cipriano E, Massaro L, Natalucci F, Capalbo G, et al. Joint involvement in systemic lupus erythematosus: from pathogenesis to clinical assessment. Semin Arthritis Rheum. 2017;47(1):53-64.

2. Danchenko N, Satia JA, Anthony MS. Epidemiology of systemic lupus erythematosus: a comparison of worldwide disease burden. Lupus. 2006;15(5):308-18.

3. Li Z, Xu D, Wang Z, Wang Y, Zhang S, Li M, et al. Gastrointestinal system involvement in systemic lupus erythematosus. Lupus. 2017;26(11):1127-38.

4. Petri M, Orbai AM, Alarcón GS, Gordon C, Merrill JT, Fortin PR, et al. Derivation and validation of the Systemic Lupus International Collaborating Clinics classification criteria for systemic lupus erythematosus. Arthritis Rheum. 2012;64(8):2677-86.

5. Tektonidou MG, Dasgupta A, Ward MM. Risk of End-Stage Renal Disease in Patients With Lupus Nephritis, 1971-2015: A systematic review and Bayesian meta-analysis. Arthritis Rheumatol. 2016;68(6):1432-41.

6. Kim H, Cho SK, Kim JW, Jung SY, Jang EJ, Bae SC, et al. An increased disease burden of autoimmune inflammatory rheumatic diseases in Korea. Semin Arthritis Rheum. 2020;50(3):526-33.

7. Bultink IEM, de Vries F, van Vollenhoven RF, Lalmohamed A. Mortality, causes of death and influence of medication use in patients with systemic lupus erythematosus vs matched controls. Rheumatology (Oxford). 2021;60(1):207-16

8. Yen EY, Shaheen M, Woo JMP, Mercer N, Li N, McCurdy DK, et al. 46-year trends in systemic lupus erythematosus mortality in the United States, 1968 to 2013: a nationwide population-based study. Ann Intern Med. 2017;167(11):777-85.
9. Hsu CY, Lin MS, Su YJ, Cheng TT, Lin YS, Chen YC, et al. Cumulative immunosuppressant exposure is associated with diversified cancer risk among 14832 patients with systemic lupus erythematosus: a nested case-control study. Rheumatology (Oxford). 2017;56(4):620-8.

10. Choi MY, Flood K, Bernatsky S, Ramsey-Goldman R, Clarke AE. A review on SLE and malignancy. Best Pract Res Clin Rheumatol. 2017;31(3):373-96.

11. Bernatsky S, Ramsey-Goldman R, Labrecque J, Joseph L, Boivin JF, Petri M, et al. Cancer risk in systemic lupus: an updated international multi-centre cohort study. J Autoimmun. 2013:42:130-5.

12. Song L, Wang Y, Zhang J, Song N, Xu X, Lu Y. The risks of cancer development in systemic lupus erythematosus (SLE) patients: a systematic review and meta-analysis. Arthritis Res Ther. 2018;20(1):270.

13. Bae EH, Lim SY, Han KD, Jung JH, Choi HS, Kim CS, et al. Systemic lupus erythematosus is a risk factor for cancer: a nationwide population-based study in Korea. Lupus. 2019;28(3):317-23.

14. Kang KY, Kim HO, Yoon HS, Lee J, Lee WC, Ko HJ, et al. Incidence of cancer among female patients with systemic lupus erythematosus in Korea. Clin Rheumatol. 2010;29(4):381-8.

15. Lee J, Lee JS, Park SH, Shin SA, Kim K. Cohort Profile: The National Health Insurance Service-National Sample Cohort (NHIS-NSC). South Korea. Int J Epidemiol. 2017;46(2):e15.

16. Hochberg MC. Updating the American College of Rheumatology revised criteria for the classification of systemic lupus erythematosus. Arthritis Rheum. 1997:40(9):1725.

17. Seo HJ, Oh $H_{1}$, Yoon SJ. A comparison of the cancer incidence rates between the national cancer registry and insurance claims data in Korea. Asian Pac Cancer Prev. 2012:13(12):6163-8.

18. Ognenovski VM, Marder W, Somers EC, Johnston CM, Farrehi JG, Selvaggi $\mathrm{SM}$, et al. Increased incidence of cervical intraepithelial neoplasia in women with systemic lupus erythematosus treated with intravenous cyclophosphamide. J Rheumatol. 2004;31(9):1763-7.

19. Chen YJ, Chang YT, Wang CB, Wu CY. Malignancy in systemic lupus erythematosus: a nationwide cohort study in Taiwan. Am J Med. 2010;123(12):1150.e1-6.

20. Chun BC, Bae SC. Mortality and cancer incidence in Korean patients with systemic lupus erythematosus: results from the Hanyang lupus cohort in Seoul. Korea. Lupus. 2005;14(8):635-8.

21. Bateman H, Yazici Y, Leff L, Peterson M, Paget SA. Increased cervical dysplasia in intravenous cyclophosphamide-treated patients with SLE: a preliminary study. Lupus. 2000;9(7):542-4.

22. Löfström B, Backlin C, Sundström C, Ekbom A, Lundberg IE. A closer look at nonHodgkin's lymphoma cases in a national Swedish systemic lupus erythematosus cohort: a nested case-control study. Ann Rheum Dis. 2007;66(12):1627-32.

23. Bashir S, Harris G, Denman MA, Blake DR, Winyard PG. Oxidative DNA damage and cellular sensitivity to oxidative stress in human autoimmune diseases. Ann Rheum Dis. 1993:52(9):659-66.

24. Lin YJ, Wan L, Huang CM, Chen SY, Huang YC, Lai CH, et al. Polymorphisms in the DNA repair gene XRCC1 and associations with systemic lupus erythematosus risk in the Taiwanese Han Chinese population. Lupus. 2009;18(14):1246-51.

25. Noble PW, Bernatsky S, Clarke AE, Isenberg DA, Ramsey-Goldman R, Hansen JE. DNA-damaging autoantibodies and cancer: the lupus butterfly theory. Nat Rev Rheumatol. 2016;12(7):429-34.

26. Löfström B, Backlin C, Pettersson T, Lundberg IE, Baecklund E. Expression of APRIL in diffuse large B cell lymphomas from patients with systemic lupus erythematosus and rheumatoid arthritis. J Rheumatol. 2011;38(9):1891-7.

27. Lyrio LD, Grassi MF, Santana IU, Olavarria VG, Gomes Ado N, CostaPinto L, et al. Prevalence of cervical human papillomavirus infection in women with systemic lupus erythematosus. Rheumatol Int. 2013;33(2):335-40.

28. Bernatsky S, Boivin JF, Joseph L, Manzi S, Ginzler E, Gladman DD, et al. Mortality in systemic lupus erythematosus. Arthritis Rheum. 2006;54(8):2550-7.

29. Thomas G, Mancini J, Jourde-Chiche N, Sarlon G, Amoura Z, Harlé JR, et al. Mortality associated with systemic lupus erythematosus in France assessed by multiple-cause-of-death analysis. Arthritis Rheumatol. 2014;66(9):2503-11.

30. Choi HK, Nguyen US, Niu J, et al. Selection bias in rheumatic disease research. Nat Rev Rheumatol 2014;10:403-412. 2014/04/02. https://doi.org/ 10.1038/nrrheum.2014.36

\section{Publisher's Note}

Springer Nature remains neutral with regard to jurisdictional claims in published maps and institutional affiliations. 